\title{
Experimental Validation of Generating Two Spaced Beams with Reflectarrays by VRT
}

\author{
Alvaro Somolinos, Rafael Florencio, Iván González, José A. Encinar and Felipe Cátedra
}

\begin{abstract}
The concept of generating two spaced beams in dual-CP by variable rotation technique (VRT) is validated for first time by manufacturing and measuring. A reflectarray demonstrator has been designed, manufactured and tested to deviate $\pm 10^{\circ}$ the beam of a dual-CP multi-flared horn at $19.7 \mathrm{GHz}$. The proposed reflectarray cell for VRT is made of a conductive cross printed on a grounded dielectric. The experimental results show two adjacent beams in dual-CP maintain the correct directions in the prescribed frequency band (19.2-20.2 GHz). The results are satisfactory and validate the concept for generating two spaced beams in orthogonal CP by VRT with a single feeds.
\end{abstract}

Index Terms - reflectarray, satellite antennas, multibeam antennas, moment method.

\section{INTRODUCTION}

During last two decades, the technology for analyzing and designing reflectarray antennas has received a large impulse. Now, many mature applications can be found in the literature, [1]-[4]. Reflectarrays offer major flexibility for new antenna designs than metallic reflectors. One of the best advantages of reflectarrays is that they can be designed using microstrip layouts over flat surfaces or other surfaces. Reflectarray antennas are useful to be fabricated using a "mass production scale"

More recently, it has been demonstrated that it is possible to design and build reflectarrays with a degree of accuracy similar to the mature technology of reflector antennas. It can be found in the literature that reflectarray antennas can provide gain, efficiency, polarization purity and beam shaping features good enough for satellite applications [5][7], which demand very stringent antennas specifications.

Today dual-polarization, dual-band reflectarrays have received a great interest for multi-beam satellite antennas because this technology could reduce the number of apertures in the satellite. The work presented in [8] shows a dual-band reflectarray working at 19.7 GHz and $29.5 \mathrm{GHz}$ using two sets of stacked parallel dipoles that give two close spot beams in orthogonal lineal polarization at both frequency bands. Reflectarrays based on rotating elements have been demonstrated for generating a pencil beam in circular polarization with very low levels of cross-polarization [9]-[11].

The VRT, as shown in [12], has four interesting features for circular polarization: (a) The VRT requires that the reflection coefficients of the two ortogonal linear components are of equal magnitude and $180^{\circ}$ out of phase; (b) the reflected circular polarized waves save their polarization sense for both right handed (RH) and left handed (LH) components; (c) an additional phase is added to the phase of the reflection coefficient for circular polarization which depends on twice the rotation angle of the element, approximately; (d) the additional phase changes with rotation in opposite sense for the right and left polarizations. This additional phase change allows the design of reflectarray by generating layouts with rotated elements. We can remark than the opposite senses for the phase variation with the rotation angles for the RH and LH polarizations can permit the design of dual beams using the same layout as shown in [13]-[14]. In these last references, well optimized designs for reflectarrays conformed to parabolic surfaces are presented using VRT. The unit cell of the reflectarray is one dipole printed on a single dielectric layer, which only allows the phase adjustment for one linear field component, being the orthogonal component imposed by the dielectric thickness. The phasing capabilities of the proposed reflectarray cells are very limited and impose some constraints on the dielectric thickness, to ensure the required phase difference of 180 degrees by controlling only one field component. Although the simulation results presented in [13]-[14] are very promising, the concept of generating two spaced beams in dual-CP by VRT has not been experimentally demonstrated. In this work, we validate experimentally the concept of generating two spaced beams in dual-CP by VRT. Unlike [13]-[14], we have chosen as rotating element a dual linear polarization element which consists in a cross with thin arms. Contrary to the single dipoles proposed in [13]-[14], these elements provide roughly independent phase control of the components of the reflected electric field which are parallel to the arms of the cross. This independent phase control provides an additional degree of freedom to produce the difference of $180^{\circ}$ between the phases of the reflection coefficients required to apply the VRT. The concept of generating two spaced beams in dualCP with a single dual-polarized feed is demonstrated for first time by manufacturing and measuring a $25 \mathrm{~cm} \times 25 \mathrm{~cm}$ flat reflectarray in Kaband.

\section{COMPUTATION OF THE REQUIRED ADDITIONAL PHASE TO SEPARATE THE BEAMS IN DUAL-CP}

Figure 1 shows a two-dimensional view of a reflectarray/reflector antenna, although the formulation and the results are presented for 3D curved surface. The reflectarray or reflector surface is indicated by the solid line. This surface can be flat or curved. Point $S$ indicates the position of the center of phase of the feed, point $R$ is the reflection point in the reflectarray/reflector surface of the ray-path that goes from the source, $S$, to a point $\mathrm{P}$ in the aperture plane. Lengths of path segments SR and RP are $\mathrm{d} 1$ and $\mathrm{d} 2$, respectively. We will refer to this antenna as the "Primitive Antenna". Its radiation pattern will be computed considering the magnetic equivalent current $\mathrm{M}_{\mathrm{T}}(\mathrm{x}, \mathrm{y})$ at 
point $P$ in the aperture plane $A^{\prime}$, which will be the $X Y$ plane in the coordinate reference system.

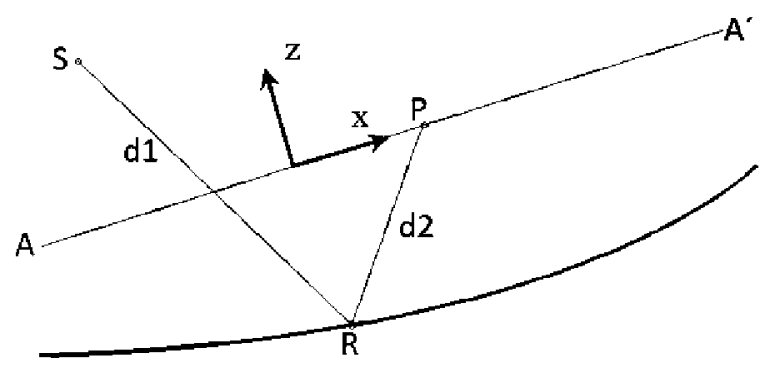

Figure 1. Scheme of reflectarray/reflector antenna. by:

The phase delay $\emptyset_{\mathrm{T}}(\mathrm{x}, \mathrm{y})$ of the magnetic current at point $\mathrm{P}$ is given

$$
\emptyset_{\mathrm{T}}(\mathrm{x}, \mathrm{y})=\emptyset_{1}(\mathrm{x}, \mathrm{y})+\emptyset_{2}(\mathrm{x}, \mathrm{y})+\emptyset_{\mathrm{R}}(\mathrm{x}, \mathrm{y})
$$

where $\emptyset_{1}$ and $\emptyset_{2}$ are the phase delay introduced by the propagation along path segments $\mathrm{SR}$ and $\mathrm{RP}$, respectively:

$$
\begin{aligned}
& \emptyset_{1}(\mathrm{x}, \mathrm{y})=\mathrm{k}_{\mathrm{o}} \cdot \mathrm{d}_{1}(\mathrm{x}, \mathrm{y}, \mathrm{z}=0) \\
& \emptyset_{2}(\mathrm{x}, \mathrm{y})=\mathrm{k}_{\mathrm{o}} \cdot \mathrm{d}_{2}(\mathrm{x}, \mathrm{y}, \mathrm{z}=0)
\end{aligned}
$$

where $k_{0}$ is the wavenumber at the working frequency. The phase delay $\emptyset_{\mathrm{R}}$ is introduced by the reflection coefficient of the reflectarray cell at point R. When the Primitive Antenna is a reflector, $\emptyset_{\mathrm{R}}=\pi$ radians. To compute the radiation pattern of the Primitive Antenna we will apply the Equivalence Principle [15] in the aperture plane shown in Figure. 1. We will consider for this computation the coordinate axes associated with this plane, such that its z-axis is perpendicular to the plane and its positive semi-axis is in the upper semi-space on Figure 1. The equivalent magnetic current can be expressed at point $\mathrm{P}$ of coordinates $(\mathrm{x}, \mathrm{y})$ of this system as follows:

$$
\mathrm{M}_{\mathrm{T}}(\mathrm{x}, \mathrm{y})=\mathrm{M}_{0}(\mathrm{x}, \mathrm{y}) \exp \left(-\mathrm{j} \phi_{\mathrm{T}}(\mathrm{x}, \mathrm{y})\right)
$$

where $\mathrm{M}_{0}(\mathrm{x}, \mathrm{y})$ takes into account the amplitude and phase variation of the complex amplitudes of the $\mathrm{x}$ and $\mathrm{y}$ components of the electric field generated by the feed located at point $\mathrm{S}$. In this work, circular polarized feed will be considered.

We can apply a Fourier Transform to compute the un-normalized radiation pattern $\mathrm{D}\left(\mathrm{k}_{\mathrm{x}}, \mathrm{k}_{\mathrm{y}}\right)$ for directions not so far from the mentioned z-axis:

$$
\mathrm{D}\left(\mathrm{k}_{\mathrm{x}}, \mathrm{k}_{\mathrm{y}}\right)=\int_{S \mathrm{p}} \mathrm{M}_{\mathrm{T}}(\mathrm{x}, \mathrm{y}) \exp \left(-\mathrm{jk}_{\mathrm{x}} \mathrm{x}-\mathrm{j} \mathrm{k}_{\mathrm{y}} \mathrm{y}\right) \mathrm{dxdy}
$$

where $\left(\mathrm{k}_{\mathrm{x}}, \mathrm{ky}\right)$ are the spectral angular coordinates

$$
\begin{aligned}
& \mathrm{k}_{\mathrm{x}}=\mathrm{k}_{o} \cos (\varphi) \sin (\theta) \\
& \mathrm{k}_{\mathrm{y}}=\mathrm{k}_{o} \sin (\varphi) \sin (\theta)
\end{aligned}
$$

$(\theta, \varphi)$ are the spherical angular coordinates and Sp indicates the aperture surface of the Primitive Antenna in the aperture plane $\mathrm{AA}^{\prime}$ where the Equivalence Principle [15] is applied.

Depending of the design parameters, the pattern $\mathrm{D}\left(\mathrm{k}_{\mathrm{x}}, \mathrm{k}_{\mathrm{y}}\right)$ of the Primitive Antenna can have a collimated narrow beam, a shaped conformed beam or any other form.

By adding in the reflectarray or reflector surface a layout of variable rotation (VR) elements, we can obtain for a given circular polarization $(\mathrm{RH})$, that we will call co-polar sense, the following expression for the phase of the reflection coefficient:

$$
\emptyset_{\mathrm{R}}^{\mathrm{RH}}(\mathrm{x}, \mathrm{y})=\emptyset_{\mathrm{R}}(\mathrm{x}, \mathrm{y})+\mathrm{k}_{\mathrm{x}}^{\prime} \mathrm{x}+\mathrm{k}_{\mathrm{y}}^{\prime} \mathrm{y}
$$

where $\mathrm{k}_{\mathrm{x}}^{\prime}$ and $\mathrm{k}_{\mathrm{y}}^{\prime}$ are the spectral angular coordinates for the desired direction of the beam in $\mathrm{CP}\left(\theta^{\prime}, \varphi^{\prime}\right)$

$$
\begin{aligned}
& \mathrm{k}_{\mathrm{x}}^{\prime}=\mathrm{k}_{\mathrm{o}} \cos \left(\varphi^{\prime}\right) \sin \left(\theta^{\prime}\right) \\
& \mathrm{k}_{\mathrm{y}}^{\prime}=\mathrm{k}_{\mathrm{o}} \sin \left(\varphi^{\prime}\right) \sin \left(\theta^{\prime}\right)
\end{aligned}
$$

with the new reflection phase we obtain for the co-polar circular polarization the following un-normalized radiation pattern:

$D^{R H}\left(k_{x}, k_{y}\right)=\int_{S p} M_{T}(x, y) \exp \left(-j\left(k_{x}-k_{x}^{\prime}\right) x-j\left(k_{y}-k_{y}^{\prime}\right) y\right) d x d y$

It can be seen that the new and original patterns are related by

$$
\mathrm{D}^{\mathrm{RH}}\left(\mathrm{k}_{\mathrm{x}}, \mathrm{k}_{\mathrm{y}}\right)=\mathrm{D}\left(\mathrm{k}_{\mathrm{x}}-\mathrm{k}_{\mathrm{x}}^{\prime}, \mathrm{k}_{\mathrm{y}}-\mathrm{k}_{\mathrm{y}}^{\prime}\right)
$$

We can appreciate that the new radiation pattern is similar to the original one except for a shift in the angular spectral space given by the vector

$$
\mathrm{k}^{\mathrm{RH}}=\mathrm{k}_{\mathrm{x}}^{\prime} \hat{\mathrm{x}}+\mathrm{k}_{\mathrm{y}}^{\prime} \hat{\mathrm{y}}
$$

We know from the theory of VRT that for the orthogonal circular polarization $(\mathrm{LH})$, the phase of the reflection coefficient is given by:

$$
\emptyset_{\mathrm{R}}^{\mathrm{LH}}(\mathrm{x}, \mathrm{y})=\emptyset_{\mathrm{R}}(\mathrm{x}, \mathrm{y})-\mathrm{k}_{\mathrm{x}}^{\prime} \mathrm{x}-\mathrm{k}_{\mathrm{y}}^{\prime} \mathrm{y}
$$

and the radiation pattern has the original radiation pattern with a shift of the spectral coordinates opposite to the co-polar case

$$
\mathrm{D}^{\mathrm{LH}}\left(\mathrm{k}_{\mathrm{x}}, \mathrm{k}_{\mathrm{y}}\right)=\mathrm{D}\left(\mathrm{k}_{\mathrm{x}}+\mathrm{k}_{\mathrm{x}}^{\prime}, \mathrm{k}_{\mathrm{y}}+\mathrm{k}_{\mathrm{y}}^{\prime}\right)
$$

and now the spectral shift vector is given by $\mathrm{k}^{\mathrm{LH}}$

$$
\mathrm{k}^{\mathrm{LH}}=-\mathrm{k}^{\mathrm{RH}}=-\mathrm{k}_{\mathrm{x}}^{\prime} \hat{\mathrm{x}}-\mathrm{k}_{\mathrm{y}}^{\prime} \hat{\mathrm{y}}
$$

From the previous development, we can see that a dual beam antenna can be developed using a Primitive Antenna with a reflectarray/reflector surface, flat or curved, fed by a dual circular polarized source by adding a layout of VR elements. According with equations (9), (10) and (12), (13), if the Primitive Antenna generate a same pencil beam in a given direction for each circular component, the new antenna generate two spaced beams. Each beam has been displaced opposite spectral shift vectors with respect to the original beam.

Now we will relate the shifts on the real space spherical angular coordinates with the angular spectral coordinates. We start assuming that the reference axis of the main beam of the Primitive Antenna is given by the spherical angular coordinates $\left(\theta_{\mathrm{m}}, \varphi_{\mathrm{m}}\right)$. The spectral angular coordinates of this axis, $\left(\mathrm{k}_{\mathrm{mx}}, \mathrm{k}_{\mathrm{my}}\right)$ can be obtained from:

$$
\begin{aligned}
& \mathrm{k}_{\mathrm{mx}}=\mathrm{k}_{\mathrm{o}} \cos \left(\varphi_{\mathrm{m}}\right) \sin \left(\theta_{\mathrm{m}}\right) \\
& \mathrm{k}_{\mathrm{my}}=\mathrm{k}_{\mathrm{o}} \sin \left(\varphi_{\mathrm{m}}\right) \sin \left(\theta_{\mathrm{m}}\right)
\end{aligned}
$$

By introducing the layout of VR elements the main axis changes its orientation and now its direction for the co-polar circular component is described by the spherical angles $\left(\theta_{\mathrm{m}}+\Delta \theta, \varphi_{\mathrm{m}}+\Delta \varphi\right)$, related with the corresponding spectral coordinates $\left(\mathrm{k}_{\mathrm{mx}}+\mathrm{k}_{\mathrm{x}}^{\prime}, \mathrm{k}_{\mathrm{my}}+\mathrm{k}_{\mathrm{y}}^{\prime}\right)$ as follows:

$$
\begin{aligned}
& \mathrm{k}_{\mathrm{mx}}+\mathrm{k}_{\mathrm{x}}^{\prime}=\mathrm{k}_{\mathrm{o}} \cos \left(\varphi_{\mathrm{m}}+\Delta \varphi\right) \sin \left(\theta_{\mathrm{m}}+\Delta \theta\right) \\
& \mathrm{k}_{\mathrm{my}}+\mathrm{k}_{\mathrm{y}}^{\prime}=\mathrm{k}_{\mathrm{o}} \sin \left(\varphi_{\mathrm{m}}+\Delta \varphi\right) \sin \left(\theta_{\mathrm{m}}+\Delta \theta\right)
\end{aligned}
$$

By combining equations (14) and (15) we found:

$$
\begin{gathered}
\Delta \theta=\operatorname{asin}\left(\sqrt{\frac{\mathrm{k}_{0}^{2} \sin \left(\theta_{\mathrm{m}}\right)^{2}+\mathrm{k}_{\mathrm{x}}^{\prime 2}+\mathrm{k}_{\mathrm{y}}^{\prime 2} \mp 2 \mathrm{k}_{\mathrm{o}} \sin \left(\theta_{\mathrm{m}}\right)\left(\mathrm{k}_{\mathrm{x}}^{\prime} \cos \left(\varphi_{\mathrm{m}}\right)+\mathrm{k}_{\mathrm{y}}^{\prime} \sin \left(\varphi_{\mathrm{m}}\right)\right)}{\mathrm{k}_{0}^{2}}}\right)- \\
\theta_{\mathrm{m}}
\end{gathered}
$$

The previous expressions can be particularized to some interesting cases, for instance when the beam steering is in $\mathrm{XZ}$ plane: 


$$
\begin{aligned}
\varphi_{\mathrm{m}} & =0 \\
\mathrm{k}_{\mathrm{y}}^{\prime} & =0
\end{aligned}
$$

In this case we have that

$$
\begin{gathered}
\Delta \theta=\operatorname{asin}\left(\frac{\mathrm{k}_{0} \sin \left(\theta_{\mathrm{m}}\right) \mp \mathrm{k}_{\mathrm{x}}^{\prime}}{\mathrm{k}_{\mathrm{o}}}\right)-\theta_{\mathrm{m}} \\
\Delta \varphi=0
\end{gathered}
$$

A particular subcase of the previous case is when expressions (17) are satisfied but also we have that

$$
\begin{gathered}
\mathrm{k}_{\mathrm{x}}^{\prime} \ll \mathrm{k}_{0} \\
\theta_{\mathrm{m}} \rightarrow 0
\end{gathered}
$$

In this case we have for $\Delta \theta$;

$$
\Delta \theta=\frac{\mathrm{k}_{\mathrm{o}} \theta_{\mathrm{m}} \mp \mathrm{k}_{\mathrm{x}}^{\prime}}{\mathrm{k}_{\mathrm{o}}}-\theta_{\mathrm{m}}=\mp \frac{\mathrm{k}_{\mathrm{x}}^{\prime}}{\mathrm{k}_{\mathrm{o}}}
$$

According with (20) for obtaining a tilt of $\Delta \theta$ of the main beam with $\Delta \varphi=0$, the rotating element shall introduce a phase delay slope of

$$
\mathrm{k}_{\mathrm{x}}^{\prime}=\mp \mathrm{k}_{\mathrm{o}} \Delta \theta
$$

This phase delay slope is achieved by imposing that the unit cell element to be conformed on the reflectarray/reflector surface at coordinates $(\mathrm{x}, \mathrm{y}, \mathrm{z})$ introduces a phase delay given by

$$
\emptyset_{\mathrm{VR}}(\mathrm{x}, \mathrm{y}, \mathrm{z})=\mp \mathrm{k}_{\mathrm{o}} \Delta \theta \mathrm{x}+\mathrm{C}
$$

where $\mathrm{C}$ is a constant value. In this paper we have considered the flat reflectarray shown in Figure 2. This antenna has been designed, built and measured with the purpose of experimental validation of the concept of separation of the beams in dual-CP by VRT. The real circular polarized radiation pattern of the horn will be approximated by $\cos ^{20}$ $(\theta)$ function. The feed is located in $(-0.1108,0.0,0.2384)(\mathrm{m})$ and it is oriented to point $(-0.0145,0,0)(\mathrm{m})$ where we located a cross with its arms parallel to the coordinated axes. The Snell angle that in this case gives the direction of the main beam of the primitive antenna is of $22^{\circ}$.

The reflectarray has been built imposing a phase difference between contiguous rows of $28.55^{\circ}$, in order to get a deviation of the beam of about $10^{\circ}$ relative the Primitive Antenna. In particular applying expressions (18) we obtain for the RHCP a deviation of the beam of $\Delta \theta$ $=+10.24^{\circ}$ and for the LHCP a deviation of the beam of $\Delta \theta=-9.54^{\circ}$.

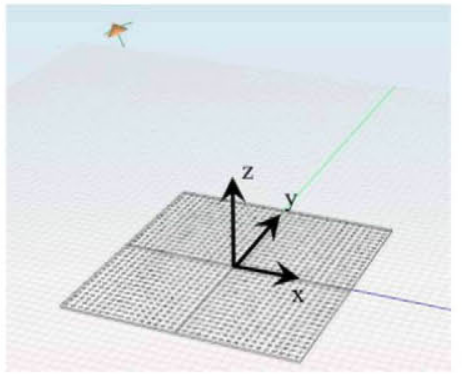

Figure 2. Flat squared reflectarray with a side of $25 \mathrm{~cm}$. The reflectarray center is in the origin of coordinated and the focus has been located at $(-0.1108,0.0$, 0.2384 ) in meters.

\section{PROPOSED UNIT CELL FOR THE VARIABLE ROTATION TECHNIQUE}

We consider a normally incident RHCP plane wave on a periodic array with the unit cell shown in Figure. 3 . The unit cell consist of a cross embedded in the interface of the two dielectric layers shown in Figure $3 b$. The plane wave is propagating in the $-\hat{z}^{\prime}$ direction, with electric field expressed as:

$$
\vec{E}_{\text {inc }}^{\mathrm{RHCP}}=E_{0}\left(\hat{x}^{\prime}+j \hat{y}\right) e^{j k_{0} z^{\prime}}
$$

where $\hat{x}^{\prime}$ and $\hat{y}^{\prime}$ are the unitary vector along the $x^{\prime}$ and $y^{\prime}$ axis shown in Figure. 3a.

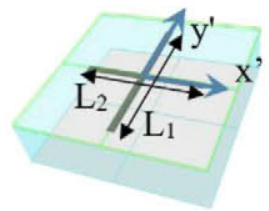

(a)

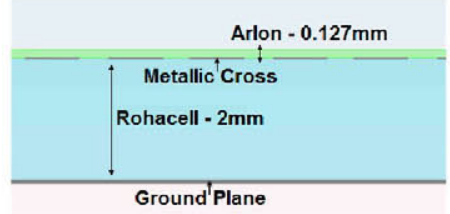

(b)
Figure 3. (a) Unit cell defined by a cross embedded between the two dielectric layers and located in plane parallel to the reflectarray ground plane. (b) Dielectric layers for unit cell. The permittivity of dielectric is $\varepsilon_{\mathrm{r}}=1.12$ with a loss tangent of $\tan \delta_{\mathrm{r}}=0.002$ for the Rohacell and $\varepsilon_{\mathrm{r}}=2.3$ and $\tan \delta_{\mathrm{r}}=0.002$ for the Arlon.

The electric field of the reflected electromagnetic wave can be expressed as a sum of two circularly polarized waves that propagate in the $\hat{z}^{\prime}$ positive direction, according to [9] and [12].

$$
\begin{aligned}
\vec{E}_{\mathrm{ref}}= & \frac{1}{2} e^{j 2 \alpha} E_{0}\left(R_{x^{\prime} x^{\prime}}-R_{y^{\prime} y^{\prime}}\right)\left(\hat{x}^{\prime}-j \hat{y}\right) e^{-j k_{0} z^{\prime}}+ \\
& \frac{1}{2} E_{0}\left(R_{x^{\prime} x^{\prime}}+R_{y^{\prime} y^{\prime}}\right)\left(\hat{x}^{\prime}+j \hat{y}\right) e^{-j k_{0} z^{\prime}}
\end{aligned}
$$

where $R_{x^{\prime} x^{\prime}}$ and $R_{y^{\prime} y^{\prime}}$ are the reflection coefficients for the field components oriented in the directions of the crossed dipoles in directions of the $x^{\prime}$ and $y^{\prime}$ axes, respectively (see Figure 3 ).

The first term of the sum in (24) is the reflected circularly polarized wave with the same direction of rotation as the incident field (RHCP), since the reflected wave propagates the $\hat{z}$ direction and the incident waves propagates the $-\hat{z}$ direction. The phase of this wave is proportional to the rotation angle $\alpha$ of the cross. The second component is the reflected circularly polarized wave opposite to that of the incident wave. According to (24), the phase of the reflected RHCP wave is proportional to $2 \alpha$ when the following condition is fulfilled:

$$
R_{x^{\prime} x^{\prime}}=-R_{y^{\prime} y^{\prime}}
$$

In other words, it is necessary to provide a differential phase shift of 180 degrees between the reflection components of the orthogonal field in the directions of the cross arms ( $x^{\prime}$ and $\left.y^{\prime}\right)$. Note that when the incidence is given in on a plane containing one of the cross arms, the cross-polar reflection coefficients are zero $\left(R_{x^{\prime} y^{\prime}}=R_{y^{\prime} x^{\prime}}=0\right)$ because of the symmetry. Therefore, we need that the reflection coefficient matrix for lineal polarization should satisfy as close as possible to the following matrix equation:

$$
\left[\begin{array}{ll}
\mathrm{R}_{\mathrm{x} / \mathrm{x} \prime} & \mathrm{R}_{\mathrm{x} / \mathrm{y}^{\prime}} \\
\mathrm{R}_{\mathrm{y} / \mathrm{x} \prime} & \mathrm{R}_{\mathrm{y} / \mathrm{y} \prime}
\end{array}\right]=\left[\begin{array}{cc}
1 & 0 \\
0 & -1
\end{array}\right] \exp \left(-\mathrm{j} \emptyset_{\mathrm{x} \prime}\right)
$$

where $\emptyset_{\mathrm{x},}$ is the phase of the $\mathrm{R}_{\mathrm{x}}{ }^{\prime} \mathrm{x}^{\prime}$ reflection coefficient.

When this condition is fulfilled, the reflected field is:

$$
\vec{E}_{\mathrm{ref}}^{\mathrm{RHCP}}=e^{j 2 \alpha} R_{x^{\prime} x^{\prime}} E_{0}\left(\hat{x}^{\prime}-j \hat{y}\right) e^{-j k_{0} z^{\prime}}
$$

Assuming that (26) is fulfilled, the reflection matrix for the ideal element in circular polarization is as follows:

$$
\left[\begin{array}{ll}
\mathrm{R}_{\mathrm{rr}} & \mathrm{R}_{\mathrm{lr}} \\
\mathrm{R}_{\mathrm{rl}} & \mathrm{R}_{\mathrm{ll}}
\end{array}\right]=\left[\begin{array}{cc}
\exp (2 \mathrm{j} \alpha) & 0 \\
0 & \exp (-2 \mathrm{j} \alpha)
\end{array}\right] \exp \left(-\mathrm{j} \emptyset_{\mathrm{x}^{\prime}}\right)
$$

where $r$ and 1 sub-indices denote right and left-handed circular polarization components, respectively; and $\alpha$ is the angle of rotation of the crosses. Note that when the angle of rotation is 0 , the phase of the reflected CP is the same as that $x^{\prime}$ - field $\left(\emptyset_{\mathrm{x}}\right)$. When the orthogonal $\mathrm{CP}$ is incident, the reflected field will suffer a phase-shift with opposite sign, proportional to $-2 \alpha$, as stated in [13]. On the other hand, the phase $\emptyset_{\mathrm{x} \prime}$ provides an additional degree of freedom to change the phase of the reflected $\mathrm{CP}$. 
When the phase-shift between both coefficients is not exactly $180^{\circ}$, but there is an error in the phase of $\Delta \phi$.

$$
R_{y^{\prime} y^{\prime}}=-R_{x^{\prime} x^{\prime}} e^{j \Delta \varphi}
$$

The second term of (24) will produce a cross-polar component in the reflected field.

$$
\vec{E}_{\text {ref }}^{\mathrm{LHCP}}=\frac{1}{2} E_{0} R_{x^{\prime} x^{\prime}}\left(1-e^{j \Delta \varphi}\right)\left(\hat{x}^{\prime}+j \hat{y}\right) e^{-j k_{0} z^{\prime}}
$$

Note that the phase-shift for the cross-polar component of reflected field in CP is not produced by the rotation angle $\alpha$ of the element. This cross-polar component is only produced by the error in the phase $\Delta \phi$.

The unit cell shown in Figure. 3 have been designed for obtaining very good performances for the co-polar and cross-polar components of dual beam reflectarrays build using the VRT. We have considered the same working frequency, $19.7 \mathrm{GHz}$. We consider the incident wave is forming an angle of 22 degrees with the normal vector to the cross surface. We adjust three optimized pairs for the cross arms lengths to fulfill (25). The first pair of lengths has 6.0 and $4.9 \mathrm{~mm}$ for the larger and short arms of the cross, respectively. This pair of length has been obtained optimizing the response of the cross when its arms are parallel to the cell unit side ( 0.0 degree of rotation angle). The second and third pair of lengths have been obtained optimizing the response of the cross when it is rotated 22.5 and 45.0 degrees and are given in $\mathrm{mm}$ by (6.14, $4.91)$ and $(6.24,4.92)$, respectively. We can notice that the design for rotation angles of 22.5 and 45.0 degrees is also an optimum solution for rotation angles of -22.5 and -45.0 degrees.

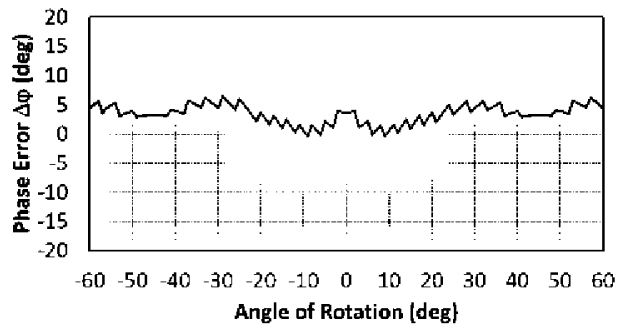

(a)

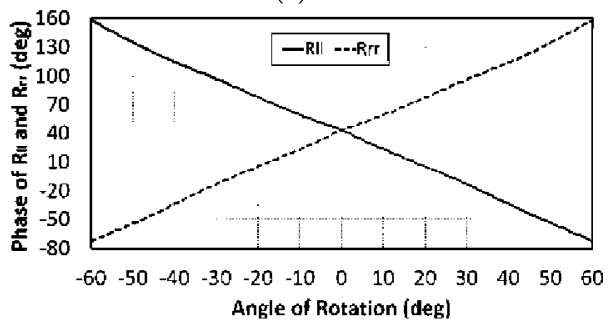

(b)

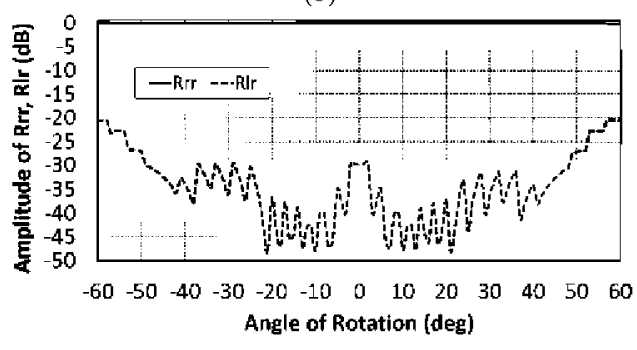

(c)

Figure 4. Results obtained at $19.7 \mathrm{GHz}$ of the optimized cell when the cross is rotated clockwise. (a) Phase error $\Delta \phi$ for the components in linear polarization, according to (28). (b) Phase of the copolar reflection coefficients for circular polarization. Again the phase for $\mathrm{R}_{\mathrm{rr}}$ increases with the rotation angle as $2 \alpha$ and the phase of the $R_{11}$ decrease with the rotation angle as $-2 \alpha$. Note that when the element isn't rotated $(\alpha=0)$ the phases for $\mathrm{rr}$ and $\mathrm{ll}$ components are identical. (c) Amplitude of the circular reflection coefficients for co-polar component $\mathrm{R}_{\mathrm{rr}}$ (solid line); and for cross-polar component $\mathrm{R}_{\mathrm{lr}}$ (dashed line).
Therefore we have five pairs of lengths which provide the required value of $180^{\circ}$ difference of phase between the copolar reflection

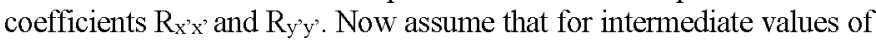
rotation angle an optimized value for the lengths of the cross arms can be obtained by mean of a lineal interpolation of the pairs of lengths.

Figure 4 shows results obtained at $19.7 \mathrm{GHz}$ of the optimized cell when the cross is rotated clockwise. These results have been obtained with the MoM formulation for infinite periodic structures [16]. Figure 4(a) shows the phase error $\Delta \phi$ for the components in linear polarization at $19.7 \mathrm{GHz}$, according to (29). These errors are very small. In the worst case, $6^{\circ}$ of phase error in the linear components will produce a level or cross-polarization of $-26 \mathrm{~dB}$, according to (30) considering $\mathrm{E}_{0}=1$. Despite this level of phase errors, in Figure 4(b) the phase curves show very linear behavior with respect of rotation angle and the slope is roughly +2 for $R_{r r}$ and -2 for $R_{11}$ reflection coefficients. The amplitude of the reflection coefficients $R_{\mathrm{rr}}$ and $\mathrm{R}_{\mathrm{lr}}$ components of the matrix of circular reflection components are shown in Figure 4(c). Similar results are obtained for reflection coefficients $\mathrm{R}_{\mathrm{Il}}$ and $\mathrm{R}_{\mathrm{rl}}$. We see that the level of the off-diagonal elements are roughly $30 \mathrm{~dB}$ below the $0 \mathrm{~dB}$ level of the diagonal elements. This fact will be very useful for obtaining reflectarrays design with low level of crosspolar radiation.

\section{DESIGN, MANUFACTURING AND TEST OF A 25-CM FLAT REFLECTARRAY DEMOSTRATOR}

A square $25 \times 25 \mathrm{~cm}$ flat reflectarray was designed, built and measured in order to experimentally validate for first time that a single CP feed can be used to generate two closely spaced beam in orthogonal CP, by applying the VRT. Although the technique can be applied to arbitrary surfaces, in this case the primary reflector is a flat plate that reflects the radiation pattern of the horn at an angle of $22^{\circ}$. By applying VRT, the two spaced beams are produced in orthogonal $\mathrm{CP}$. The geometry is shown in Figure 2 and details of its design are given in section II.

A smooth multi-flare multi-frequency conical horn antenna designed, manufactured and tested at the Universidad Politécnica de Madrid (UPM) with a diameter of $54 \mathrm{~mm}$ has been used to feed the flat reflectarray demonstrator. A commercial polarizer and diplexer have been used from the FEED-0183-1416 from VIKING. The measured gain is $18.8 \mathrm{dBi}$ and the cross-polar levels are $16 \mathrm{~dB}$ below the maximum at $19.7 \mathrm{GHz}$. The beam widths in both planes of the conical horn were roughly 18.5 and 21.4 degrees.

When the reflectarray is not introducing any phase-shift, the radiation pattern of the feed-horn will be radiated at $22^{\circ}$, according to the Snell law. The objective is to deviate $10^{\circ}$ the reflected beam in RHCP and $10^{\circ}$ for LHCP. It was computed that the phase-shift that must be implemented from one row to the next should be $28.55^{\circ}$, using RHCP. It was checked that this progressive phase distribution on the reflectarray surface produces a beam deviation of $-10^{\circ}$ in LHCP. The required rotation angles and dimensions of the crosses were obtained by a linear interpolation of the five optimized cells considering an incidence $\theta_{\text {inc }}=22^{\circ}$, $\varphi_{\text {inc }}=0^{\circ}$. The $25 \mathrm{~cm}$ flat reflectarray with the resulting dimensions and rotation angles, was simulated using the MoM software tool from newFASANT [16]

The resulting dimensions and rotation angles were used to generate the mask for photo-etching. The rotated crossed were manufactured by chemical photo-etching from a copper clad Arlon laminated $0.127 \mathrm{~mm}$ thick, of relative dielectric constant $\mathrm{er}=2.3$ and loss tangent, $\tan \delta=$ 0.0025 . The printed array was bonded to a $2-\mathrm{mm}$ thick Rohacell over a copper ground plane (FR4) and fixed with polyester screws. The flat panel and the feed chain were attached in a supporting structure to ensure the correct position of both parts.

The demonstrator was measured in the compact range anechoic chamber of UPM, see Figure 5. 


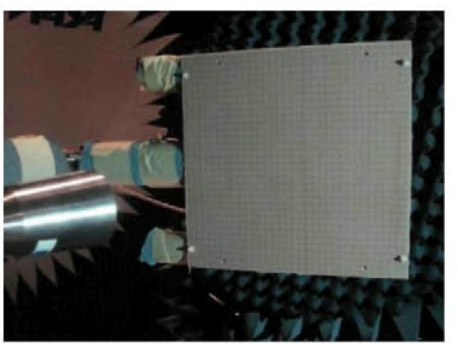

Figure 5. Demonstrator in the compact range anechoic chamber of UPM.

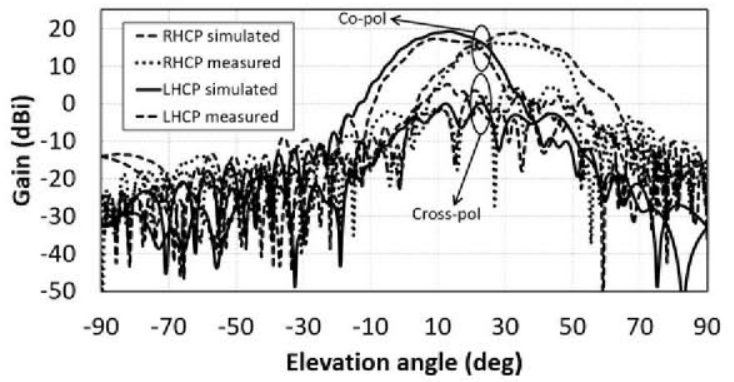

Figure 6. Simulated and measured radiation patterns for the $25 \mathrm{~cm}$ demonstrator in elevation plane, showing the beam of the horn deviated $\pm 10^{\circ}$.

The measured radiation patterns in the offset plane are compared with the simulations at $19.7 \mathrm{GHz}$ for both circular polarizations, see Figure. 6. An acceptable agreement is observed in the co-polar patterns for both RHCP and LHCP to validate the proof of concept. The patterns correspond with the main beam of the illuminating horn deviated $10^{\circ}$ for RHCP and $-10^{\circ}$ for LHCP, with respect to the specular direction $22^{\circ}$. Note that the cross-polar levels are high (15-20 dB below the maximum). These high levels of cross-polarization can be attributed to errors in the phase difference of one linear component respect to the other around $10^{\circ}$, according to equation (30). Note that the cross-polar levels of the commercial polarizer were $16 \mathrm{~dB}$. This cross-polar level increase the cross-polar radiation level of the reflectarray. The discrepancies between measurements and simulations can be attributed to phase errors, manufacturing tolerances and discrepancies in the electrical properties of the materials. For example, the simulations have been carried out using the nominal values of the materials provided by the manufacturer at $10 \mathrm{GHz}$, but an increase of the loss tangent at 19.7 $\mathrm{GHz}$ will partially justify the reduction in gain. This increment of the loss tangent may be due to the moisture present in the Rohacell. Also some phase errors are introduced in the design process. First, the direction of the main beam of the Primitive Antenna (22 degrees) is not to close to the normal direction to the reflectarray plane as assumed in expression (4). Second the analysis and design techniques assumes a fixed incidence angle $\left(\theta_{\text {inc }}=22^{\circ}, \varphi_{\text {inc }}=0^{\circ}\right)$, instead of the real incidence at each cell.

The radiation patterns have been measured in the principal planes at several frequencies $(19.2,19.7,20.2$ and $20.7 \mathrm{GHz})$, see Figure 7. The radiation patterns at different frequencies are pointing in the prescribed directions for RHCP and LHCP, but the levels of cross-polar are in the order of $15-20 \mathrm{~dB}$ below the maximum. The antenna gain at $19.2 \mathrm{GHz}$ is $2 \mathrm{~dB}$ lower, because this frequency is out of the operating frequency on the polarizer (19.4-21.2 GHz).

\section{CONCLusion}

A design technique has been proposed and experimentally validated as proof of concept for generating two closely spaced beams in orthogonal circular polarization using the variable rotation technique (VRT) in reflectarrays printed on arbitrarily shaped surfaces fed by a single dual circular polarized horn.

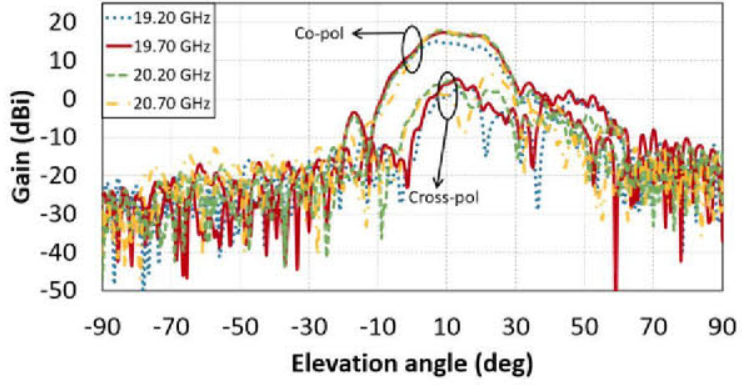

(a)

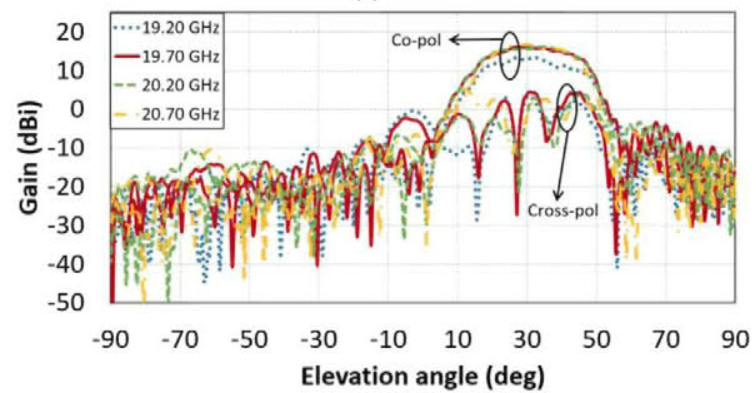

(b)

Figure 7. Measured radiation patterns in elevation plane for LHCP (a) and for RHCP (b).

A $25 \mathrm{~cm} \times 25 \mathrm{~cm}$ demonstrator has been designed, manufactured and tested to deviate $\pm 10^{\circ}$ the beam of a dual-CP multi-flared horn. The beams in RHCP and LHCP are radiated in the correct directions, in acceptable agreement with the design and simulations. The two adjacent beams in dual-CP maintain the correct directions in the prescribed frequency band (19.2-20.2 GHz). The results are satisfactory and validate the concept for generating two spaced beams in orthogonal CP with a single feed. The levels of cross-polarization are high (15-20 $\mathrm{dB}$ below the maximum), because of the high sensitivity of cross-polar radiation produced by the phase errors of the two linear components.

\section{ACKNOWLEDGMENT}

The authors would like to thank Miguel Salas Natera for providing the feed-horn and for his valuable help in the manufacturing of the antenna demonstrator.

\section{REFERENCES}

[1] D. M. Pozar, S. D. Targonski, and R. Pokuls, "A shaped-beam microstrip patch reflectarray", IEEE Trans. Antennas Propag., vol. 47, no. 7, pp. $1167-1173,1999$

[2] J. A. Encinar and J. A. Zornoza, "Three-layer printed reflectarrays for contoured beam space applications", IEEE Trans. Antennas Propag., vol. 52 , no. 5, pp. $1138-1148,2004$.

[3] J. Huang and J. A. Encinar, Reflectarray Antennas. Piscataway, NJ, USA: IEEE Press, 2008

[4] J. Shaker, M. R. Chaharmir, and J. Ethier, Reflectarray Antennas: Analysis, Design, Fabrication, and Measurement. Norwood, MA, USA: Artech House, 2014.

[5] J. A. Encinar, L. S. Datashvili, J. A. Zornoza, M. Arrebola, M. SierraCastaner, J. L. Besada-Sanmartin, H. Baier, and H. Legay, "Dualpolarization dual-coverage reflectarray for space applications", IEEE Trans. Antennas Propag., vol. 54, no. 10, pp. 2827-2837, 2006.

[6] M. Zhou, S. B. Sørensen, O. S. Kim, E. Jørgensen, P. Meincke, and O. Breinbjerg, "Direct optimization of printed reflectarrays for contoured beam satellite antenna applications", IEEE Trans. Antennas Propag., vol. 61, no. 4, pp. 1995-2004, 2013.

[7] M. Zhou, S. B. Sorensen, O. S. Kim, E. Jorgensen, P. Meincke, O. Breinbjerg, and G. Toso, "The generalized direct optimization technique 
for printed reflectarrays", IEEE Trans. Antennas Propag., vol. 62, no. 4, pp. $1690-1700,2014$.

[8] E. Martinez-de-Rioja, J. A. Encinar, R.Florencio, R.R. Boix, "Reflectarray in $\mathrm{K}$ and $\mathrm{Ka}$ Bands with Independent Beams in Each Polarization", 2016 IEEE International Symposium on Antennas and Propag., Fajardo, Puerto Rico, July 2016.

[9] J. Huang and R. J. Pogorzelski, "A Ka-band microstrip reflectarray with elements having variable rotation angles", IEEE Trans. Antennas Propag., vol. 46, no. 5, pp. 650-656, 1998.

[10] T. Smith, U. V. Gothelf, O. S. Kim, and O. Breinbjerg, "Design, manufacturing, and testing of a $20 / 30 \mathrm{GHz}$ dual-band circularly polarized reflectarray antenna in submission," IEEE Antennas Wireless Propag. Lett., vol. 12, pp. 1480-1483, 2013.

[11] R. Deng, F. Yang, S. Xu, and M. Li, "An FSS-Backed 20/30-GHz DualBand Circularly Polarized Reflectarray With Suppressed Mutual Coupling and Enhanced Performance," IEEE Transactions on Antennas and Propag., vol. 65, pp. 926-931, 2017.

[12] A. E. Martynyuk, J. I. Martinez, N.A. Martynyuk, "Spiraphase-Type Reflectarrays Based on Loaded Ring Slot Resonators", IEEE Transactions on Antennas and Propag.,vol. 52, no. 1, pp.142 - 153, 2004

[13] M. Zhou, S. B. Sorensen, "Multi-Spot Beam Reflectarrays for Satellite Telecommunication Applications in Ka-Band", (EuCAP), 2016 10th European Conference on Antennas and Propag., Davos, Switzerland, April 2016

[14] M. Zhou, S. B. Sørensen, J. R. de Lasson, N.Vesterdal, R. Jørgensen, E. Jørgensen, "Advances on High-Performance Curved Reflectarrays for Telecommunication Applications", 2017 IEEE International Symposium on Antennas and Propag., San Diego, USA, July 2017.

[15] C. A. Balanis, "Advanced Engineering Electromagnetic", John Wiley and Sons, New York, 1989.

[16] newFASANT Suite, Online: www.fasant.com 